Acta Crystallographica Section D

Biological Crystallography

ISSN 0907-4449

\title{
The structure of $\operatorname{Rv} 3717$ reveals a novel amidase from Mycobacterium tuberculosis
}

Bacterial $N$-acetylmuramoyl-L-alanine amidases are cell-wall hydrolases that hydrolyze the bond between $N$-acetylmuramic acid and L-alanine in cell-wall glycopeptides. Rv3717 of Mycobacterium tuberculosis has been identified as a unique autolysin that lacks a cell-wall-binding domain (CBD) and its structure has been determined to $1.7 \AA$ resolution by the Pt-SAD phasing method. Rv3717 possesses an $\alpha / \beta$-fold and is a zinc-dependent hydrolase. The structure reveals a short flexible hairpin turn that partially occludes the active site and may be involved in autoregulation. This type of autoregulation of activity of PG hydrolases has been observed in Bartonella henselae amidase (AmiB) and may be a general mechanism used by some of the redundant amidases to regulate cell-wall hydrolase activity in bacteria. Rv3717 utilizes its net positive charge for substrate binding and exhibits activity towards a broad spectrum of substrate cell walls. The enzymatic activity of Rv3717 was confirmed by isolation and identification of its enzymatic products by LC/MS. These studies indicate that Rv3717, an $N$-acetylmuramoyl-L-alanine amidase from M. tuberculosis, represents a new family of lytic amidases that do not have a separate $\mathrm{CBD}$ and are regulated conformationally.

\section{Introduction}

Mycobacterial diseases such as tuberculosis and leprosy remain a great threat to human life. In 2010, approximately 8.8 million incidences of and 1.1 million deaths from tuberculosis were reported, while there were an additional 0.35 million deaths from HIV-associated tuberculosis (World Health Organization, 2011). An increase in multidrug-resistant cases in both developed and developing countries in recent years, including associated mortality in HIV co-infected patients (World Health Organization, 2011), has resulted in renewed concerted efforts to search for new drugs and drug targets to combat this pathogen (Raviglione et al., 2012; Bhardwaj et al., 2011). Bacterial cell-wall biogenesis and its metabolism play crucial roles in the bacterial life cycle and in events pertaining to survival and adaptability in host tissues (Kaur et al., 2006). Consequently, the enzymes involved in cell-wall biosynthesis, including the biosynthesis of mycolic acid and peptidoglycans, are considered to be promising targets for newer therapeutic interventions in Mycobacterium tuberculosis infections (LopezMarin, 2012).

The mycobacterial cell envelope is a thick rigid structure consisting of an inner plasma membrane and an outer cell wall formed by a peptidoglycan-arabinogalactan complex. The outer lipid layer is esterified with mycolic acids that are covalently linked to the arabinogalactan layer (Hoffmann et al., 2008; Zuber et al., 2008). This arabinogalactan layer is further enclosed by a capsular layer of glycans, lipids and
Received 17 July 2013

Accepted 24 September 2013

PDB reference: $\operatorname{Rv} 3717,4 \mathrm{lq} 6$ 
proteins and serves as a barrier against the penetration of antibiotics (Brennan \& Nikaido, 1995). The unusual multilayer cell envelope limits the number of agents that are effective against tuberculosis and is also involved in the development of drug-resistant mycobacterial strains (McNeil \& Brennan, 1991; Nguyen \& Thompson, 2006; Hett \& Rubin, 2008).

Most bacteria encode a large number of redundant peptidoglycan hydrolases in their genomes that hydrolyze specific bonds in the cell-wall peptidoglycan (PG). More than 30 PG hydrolases have been identified in Escherichia coli (van Heijenoort, 2011) and Bacillus subtilis (Smith et al., 2000) on the basis of amino-acid sequence similarity. The PG hydrolases, also termed autolysins, are highly specific and have been broadly classified into three types on the basis of the bonds cleaved: $\mathrm{N}$-acetylmuramoyl-L-alanine amidases, glycan strandcleaving hydrolases and endopeptidases (Smith et al., 2000; Vollmer et al., 2008). PG hydrolases show a high degree of redundancy for the cleavage of specific bonds, underscoring their functional importance. PG hydrolases have important roles in cleavage of the septum of daughter cells, cell-wall turnover and even autolysis under specific conditions (Vollmer et al., 2008; van Heijenoort, 2011). Relatively few PG hydrolases have been identified in mycobacteria. M. phlei autolysin was one of the first cell-wall hydrolases from mycobacteria to be characterized (Li et al., 1999). A few other PG hydrolases, namely Rv3915 (CwlM), Rv2719c (cell-wall hydrolase), Rv2518c (L,D-transpeptidase), Rv1477 (RipA; endopeptidase) and Rv1478 (RipB; endopeptidase), have been identified in M. tuberculosis.

$\mathrm{N}$-Acetylmuramoyl-L-alanine amidases belonging to the PG hydrolase family hydrolyse the amide bond between $N$-acetylmuramic acid and L-alanine. $N$-Acetylmuramoyl-L-alanine amidases play a crucial role in bacterial surface growth, sporulation, germination and cell division, and bacterial pathogenesis, including adhesion to the host cells and invasion (Allignet et al., 2001; Berry et al., 1989; Rupp et al., 2001; Milohanic et al., 2001; Loessner et al., 2002; Kumar et al., 2013). Rv3915 (CwlM) is the only $N$-acetylmuramoyl-L-alanine amidase identified in M. tuberculosis to date (Deng et al., 2005) and consists of distinct PG-binding and catalytic domains. We show here that Rv3717 is an $N$-acetylmuramoyl-L-alanine amidase exhibiting cell-wall hydrolase activity and report its crystal structure at $1.7 \AA$ resolution. Rv3717 consists of a single domain and lacks the cell-wall-binding domain (CBD) usually observed in bacterial autolysins or phage endolysins. The crystal structure suggests that the overall surface charge on Rv3717 enables it to bind and hydrolyse the cell walls of several bacteria and therefore may underlie its broad spectrum of activity. In addition, the structure reveals a short flexible hairpin turn that partially occludes the active site. This hairpin turn appears to be functionally analogous to the short helical subdomain of Bartonella henselae $N$-acetylmuramoylL-alanine amidase (AmiB; Yang et al., 2012) involved in autoregulation. This type of regulation of activity of PG hydrolases may be a general mechanism used by some of the redundant amidases to regulate cell-wall hydrolase activity in bacteria.

\section{Materials and methods}

\subsection{Cloning, expression and purification of Rv3717}

The coding region of Rv3717 encoding residues 25-241 was PCR-amplified from M. tuberculosis H37Rv genomic DNA using forward and reverse primers. The forward primer introduced a Bam HI site upstream of the 25th residue, whereas the reverse primer introduced a XhoI site $3^{\prime}$ to the stop codon. The PCR product was digested with BamHI and $X h o \mathrm{I}$ and cloned in a similarly digested pET-28a (+) vector to give the expression plasmid pAMI-A. The protein encoded by pAMI-A consists of 217 residues of Rv3717 at the C-terminus with an additional 34 residues at the $\mathrm{N}$-terminus of the sequence consisting of a His tag and additional leading residues arising from cloning. The clone lacks the N-terminal 24 residues of Rv3717 encoding a predicted signal peptide. pAMI-A was transformed into E. coli C41 (DE3) strain for expression. The transformed cells were grown at $37^{\circ} \mathrm{C}$ to an $A_{600}$ of $\sim 0.6$ in Terrific Broth. The culture was then chilled on ice for $1 \mathrm{~h}$ and protein expression was induced with $0.25 \mathrm{mM}$ IPTG (isopropyl $\beta$-D-1-thiogalactopyranoside) for $12 \mathrm{~h}$ with constant shaking at room temperature $\left(25^{\circ} \mathrm{C}\right)$. Subsequently, the cells were harvested by centrifugation at $6000 \mathrm{~g}$ for $10 \mathrm{~min}$ at $277 \mathrm{~K}$. The pellet was resuspended in sonication buffer ( $20 \mathrm{~m} M$ Tris- $\mathrm{HCl} \mathrm{pH} 7.9,500 \mathrm{~m} M \mathrm{NaCl}, 5 \mathrm{~m} M$ imidazole, $1 \mathrm{~m} M$ phenylmethylsulfonyl fluoride) and was incubated with $1 \mu \mathrm{g} \mathrm{ml}^{-1}$ lysozyme on ice for $1 \mathrm{~h}$. The cells were lysed by sonication for $5 \mathrm{~min}$ and centrifuged at $29000 \mathrm{~g}$ for $30 \mathrm{~min}$ at $4{ }^{\circ} \mathrm{C}$ to remove cell debris. The His-tagged protein was purified by affinity chromatography by loading the supernatant onto nickel-nitrilotriacetate (Ni-NTA) resin (Qiagen). The column was washed with wash buffer $1(20 \mathrm{mM}$ Tris- $\mathrm{HCl} \mathrm{pH}$ 7.9, $500 \mathrm{~m} M \mathrm{NaCl}$ ) containing 5,20,50 and $100 \mathrm{~m} M$ imidazole in successive steps and the bound protein was eluted with 15 column volumes of elution buffer $(20 \mathrm{~m} M$ Tris- $\mathrm{HCl} \mathrm{pH} 7.9$, $500 \mathrm{~m} M \mathrm{NaCl}, 300 \mathrm{~m} M$ imidazole). Fractions containing significant amounts of purified protein, as determined by SDS-PAGE (12\%), were pooled. Recombinant His-tagged Rv3717 thus purified from pAMI-A ( $\mathrm{rRv} 3717-\mathrm{A})$ was dialyzed against phosphate-buffered saline (PBS) at $4^{\circ} \mathrm{C}$, concentrated to $0.50 \mathrm{mg} \mathrm{ml}^{-1}$ using an Amicon Ultra-15 (5K NMWL) concentrator (Millipore, USA) and stored at $4^{\circ} \mathrm{C}$ until further use. All activity assays described below were carried out using rRv3717-A.

\subsection{Construct for crystallization experiments}

pAMI-A was digested with BamHI and XhoI and the insert was gel-purified and cloned into a similarly digested pET28His10-Smt3 vector to give the expression vector pAMI-B. pAMI-B encodes the Rv3717 polypeptide (residues 25-241) fused to an $\mathrm{N}$-terminal $\mathrm{His}_{10}$-Smt3 tag. The recombinant construct was transformed into E. coli BL21(DE3) cells and the tagged protein was purified as described previously (Kumar et al., 2011). The N-terminal tag was then cleaved overnight using the Smt3-specific protease Ulp1 (at an Ulp1: protein ratio of $1: 500)$ at $4^{\circ} \mathrm{C}$. The tag and protease were removed over nickel-nitrilotriacetate resin (Qiagen). The 
Table 1

Data-collection statistics for the crystal structure of Rv3717.

Values in parentheses are for the outermost shell.

\begin{tabular}{ll}
\hline Space group & $I 2_{1} 2_{1} 2_{1}$ \\
Unit-cell parameters $(\AA)$ & $a=37.35, b=103.78, c=129.97$ \\
Wavelength $(\AA)$ & 1.0714 \\
Resolution $(\AA)$ & $30.91-1.68(1.77-1.68)$ \\
$R_{\text {merge }} \dagger(\%)$ & $5.4(29.1)$ \\
$R_{\text {p.i.m. }} \ddagger$ & $0.026(0.149)$ \\
$R_{\text {anom }} \S$ & $0.035(0.109)$ \\
$\langle I / \sigma(I)\rangle$ & $25.1(7.3)$ \\
Average multiplicity & $9.4(9.0)$ \\
Completeness $(\%)$ & $100(99.9)$ \\
Anomalous completeness $(\%)$ & $100(99.8)$
\end{tabular}

$\dagger R_{\text {merge }}=\sum_{h k l} \sum_{i}\left|I_{i}(h k l)-\langle I(h k l)\rangle\right| / \sum_{h k l} \sum_{i} I_{i}(h k l)$, where $\langle I(h k l)\rangle$ is the mean intensity of symmetry-related reflections $I_{i}(h k l)$. $\ddagger R_{\text {p.i.m. }}=\sum_{h k l}[1 /[N(h k l)-1]]^{1 / 2}$ $\times \sum_{i}\left|I_{i}(h k l)-\langle I(h k l)\rangle\right| / \sum_{h k l} \sum_{i} I_{i}(h k l) . \quad \S R_{\text {anom }}=\sum_{h k l}|\langle(I+)\rangle-\langle(I-)\rangle| /$ $\sum_{h k l}[\langle(I+)\rangle+\langle(I-)\rangle]$.

removal of the Smt3 tag leaves one serine residue at the $\mathrm{N}$-terminus of the purified protein. The recombinant protein thus purified from pAMI-B (rRv3717-B) was dialysed in low-salt buffer (20 m $M$ HEPES pH 7.4, $50 \mathrm{~m} M \mathrm{KCl}, 0.1 \%$ $\beta$-mercaptoethanol), concentrated using Amicon Ultra $3 \mathrm{kDa}$ molecular-weight cutoff filter units and stored at $4^{\circ} \mathrm{C}$ until further use. $r R v 3717-\mathrm{B}$ was used in all crystallization experiments described here.

\subsection{Crystallization of Rv3717}

Crystals of wild-type rRv3717-B were obtained by the sitting-drop vapour-diffusion method by mixing $2 \mu$ l protein $\left(13 \mathrm{mg} \mathrm{ml}^{-1}\right)$ with $1.5 \mu \mathrm{l}$ reservoir solution $(50 \mathrm{mM}$ Tris- $\mathrm{HCl}$ $\mathrm{pH}$ 7.5, $1.6 \mathrm{M}$ ammonium sulfate, $10 \mathrm{mM} \mathrm{MgCl}_{2}$ ) at $25^{\circ} \mathrm{C}$. Crystals appeared in $2 \mathrm{~d}$ and were allowed to grow for a further two weeks at the same temperature. Crystals of rRv3717-B were soaked for $2 \mathrm{~h}$ in $10 \mathrm{~m} M$ potassium tetrachloroplatinate (II) diluted in cryoprotectant (reservoir solution with $20 \%$ ethylene glycol) and flash-cooled in liquid nitrogen until data collection.

\subsection{Data collection and data processing}

The Pt-soaked crystal was subjected to a fluorescence scan under an attenuated beam at the Pt $K$ edge on beamline BM14 at the ESRF, Grenoble and the data were analysed with CHOOCH (Evans \& Pettifer, 2001). Diffraction data were collected from the same crystal at the Pt peak wavelength of $1.0714 \AA$ A. The data were processed using MOSFLM (Leslie \& Powell, 2007) and were scaled using SCALA within the CCP4 package (Potterton et al., 2003; Winn et al., 2011). The crystal belonged to space group $I 2_{1} 2_{1} 2_{1}$ and contained one molecule in the crystallographic asymmetric unit. Data-collection and refinement statistics are summarized in Table 1.

\subsection{Structure determination and refinement}

The structure of Rv3717 was solved by the singlewavelength anomalous dispersion (SAD) phasing method with data collected at the peak energy of the X-ray absorption spectrum of platinum using the Auto-Rickshaw automated
Table 2

Model-refinement statistics.

Values in parentheses are for the highest resolution shell.

\begin{tabular}{|c|c|}
\hline Resolution $(\AA)$ & $29.52-1.68(1.74-1.68)$ \\
\hline Protein residues built/total residues & 213/216 \\
\hline Total protein atoms & 1620 \\
\hline Total waters & 191 \\
\hline$R_{\text {work }}^{\dagger}$ & $0.160(0.170)$ \\
\hline$R_{\text {free }} \neq$ & $0.184(0.193)$ \\
\hline \multicolumn{2}{|l|}{ Average $B$ factors $\left(\AA^{2}\right)$} \\
\hline Protein atoms & 19.9 \\
\hline Water molecules & 36.9 \\
\hline Wilson $B$ factor $\left(\AA^{2}\right)$ & 21.5 \\
\hline \multicolumn{2}{|l|}{ R.m.s.d. from ideal values§ } \\
\hline Bond lengths $(\AA)$ & 0.010 \\
\hline Bond angles $\left({ }^{\circ}\right)$ & 1.0 \\
\hline \multicolumn{2}{|l|}{ Ramachandran plot $\boldsymbol{T}$, residues in } \\
\hline Most favoured region (\%) & 97.63 \\
\hline Disallowed region (\%) & 0.0 \\
\hline PDB code & $4 \mathrm{lq} 6$ \\
\hline
\end{tabular}

$\dagger R_{\text {work }}=\sum_{h k \mid}|| F_{\text {obs }}|-| F_{\text {calc }}|| / \sum_{h k l}\left|F_{\text {obs }}\right|$, where $\left|F_{\text {obs }}\right|$ is the observed structure-factor amplitude and $\left|F_{\text {calc }}\right|$ is the calculated structure-factor amplitude. $\ddagger R_{\text {free }}$ is the $R$ factor based on $5 \%$ of the data that were excluded from refinement. $\$$ As per the standard amino-acid dictionary of Engh \& Huber (1991). - From the MolProbity server (Chen et al., 2010).

structure-determination platform (Panjikar et al., 2005, 2009). Further model building of the solution obtained was carried out in Coot (Emsley et al., 2010) and refinement was performed using REFMAC5 (Murshudov et al., 2011) and BUSTER v.2.10.0 (Bricogne et al., 2011) with TLS refinement (Painter \& Merritt, 2006; residues 2-214). The final coordinates were validated using MolProbity (Chen et al., 2010). Details of the model building and refinement are given in Table 2. The surface electrostatic potential was calculated using APBS (Baker et al., 2001), while the net charge on the protein was estimated as the difference between (Lys + Arg) and (Asp + Glu) residues as described previously (Low et al., 2011); the zinc ion was included in the net charge calculation with a value of $Z=+2$.

The coordinates and structure factors of Rv3717 have been deposited in the PDB with accession code 4lq6.

\subsection{Activity of $r R v 3717-A$}

The role of rRv3717-A in 'autolysis' was indirectly tested by investigating the activity of the purified protein towards the mycobacterial cell wall of a live $M$. smegmatis culture. M. smegmatis was grown to an $A_{600}$ of 1.0 and $1 \mathrm{ml}$ of the culture was incubated with increasing concentrations of purified rRv3717-A $\left(1,25\right.$ or $50 \mu \mathrm{g}$ protein) in $1 \times \mathrm{PBS}$ at $37^{\circ} \mathrm{C}$. The $A_{600}$ was monitored for $200 \mathrm{~min}$ (without shaking) and the experiment was performed in triplicate. BSA $(50 \mu \mathrm{g})$ was used as a negative protein control, PBS was used as a buffer control and lysozyme $(50 \mu \mathrm{g})$ was used as a positive control to confirm lysis.

\subsection{Activity assay with metal ions}

The dependence of the activity of rRv3717-A on metal ions was tested using heat-killed $M$. smegmatis cells. $5 \mathrm{nmol}$ purified rRv3717-A in $20 \mathrm{~m} M$ potassium phosphate buffer was 
incubated with $1 \mathrm{ml}$ heat-killed $M$. smegmatis cells (previously grown to an $A_{600}$ of 1.5-2.0 and boiled in a water bath for $30 \mathrm{~min}$ ). The assay could not be performed by depleting the presence of residual metal ions from the protein using EDTA, as the protein precipitated during dialysis with the metal-ion chelator. Therefore, molar excesses of different metal ions $\left(\mathrm{Zn}^{2+}, \mathrm{Cu}^{2+}, \mathrm{Co}^{2+}\right.$ and $\left.\mathrm{Mg}^{2+}\right)$ at various concentrations were used for the assay (i.e. 0, 1, 10, 100 and $1000 \mu M$ ). The density of the cell suspension was monitored every minute by the $A_{600}$ for $4 \mathrm{~h}$ at $37^{\circ} \mathrm{C}$ using a Cary $100 \mathrm{UV}-$ Vis spectrophotometer. In the same assay, $5 \mathrm{nmol}$ purified lysozyme was used as a positive control and $5 \mathrm{nmol}$ BSA in the absence and presence of different metal ions was used as a negative control.

\subsection{Zymography}

In situ zymography was performed as described previously (Jayaswal et al., 1990) with minor modifications. Briefly, 12\% SDS-PAGE gels were prepared containing $0.5 \%$ heat-killed cells of different bacterial species (Paenibacillus sp., Bordetella avium, E. coli DH5 $\alpha$, Enterobacter aerogenes, Lactobacillus acidophilus, Bacillus thuringiensis, B. pumilus, B. subtilis and E. coli W3110) as substrates. $4 \mu \mathrm{g}(143 \mathrm{pmol})$ rRv3717-A was loaded in each lane of the gel. Following electrophoresis, the gels were washed twice in $50 \mathrm{ml}$ Milli-Q water with gentle stirring for $30 \mathrm{~min}$ and were then incubated at $37^{\circ} \mathrm{C}$ with $200 \mathrm{ml}$ renaturation buffer $(50 \mathrm{mM}$ Tris- $\mathrm{HCl} \mathrm{pH}$ 7.9, $200 \mathrm{mM}$ $\mathrm{NaCl}, 5 \mathrm{mM} \mathrm{CaCl}_{2}, 2 \mu \mathrm{m} \mathrm{ZnCl}, 1 \%$ Triton-X) for $12-16 \mathrm{~h}$ with gentle shaking (Buist et al., 1995). The zymographs were then rinsed with Milli-Q water and the gels were stained with $0.01 \% \mathrm{KOH}$ and $0.1 \%$ methylene blue for $30 \mathrm{~min}$ and washed

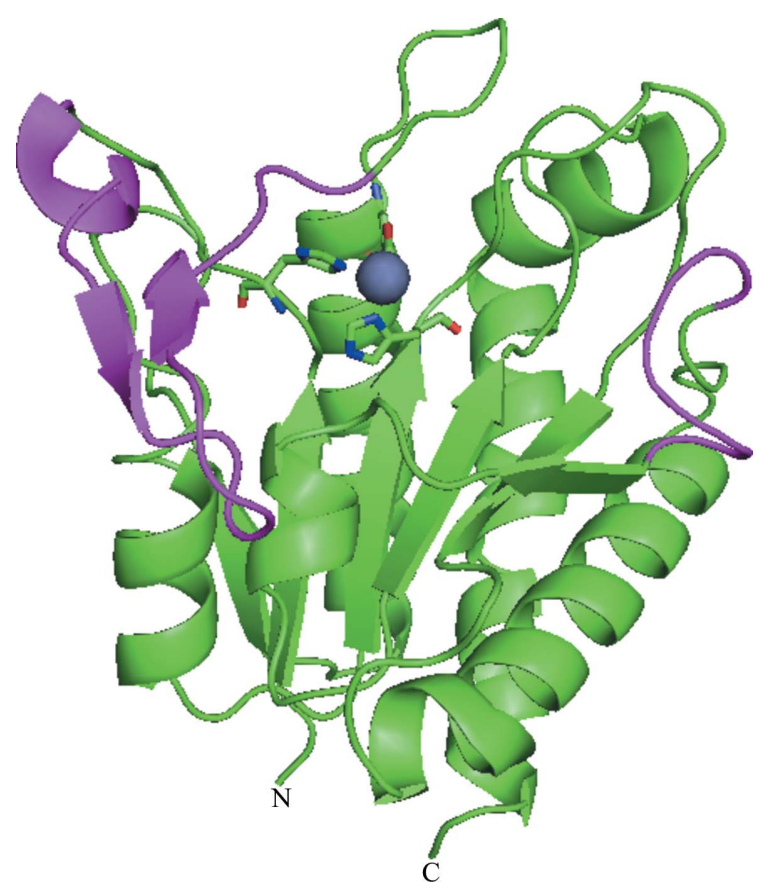

Figure 1

Overall structure of rRv3717-B indicating a single-domain structure for this amidase. The unique ${ }^{16} \beta$-hairpin ${ }^{36}$ and ${ }^{148}$ loop $^{154}$ are shown in magenta. A bound zinc ion (sphere) marks the catalytic centre of the metalloenzyme. The N- and C-termini are labelled. with Milli-Q water. Lysozyme $(4 \mu \mathrm{g})$ and bovine serum albumin (BSA; $4 \mu \mathrm{g}$ ) were used as positive and negative controls, respectively. Clear bands on an opaque background indicated hydrolysis. The molecular masses of the clarified bands were determined by comparison with pre-stained molecular-weight standards electrophoresed on the same gel.

\subsection{LC/MS analysis of enzymatic products of the $\mathrm{N}$-acetylmuramoyl-L-alanine amidase activity of rRv3717-A}

The activity of rRv3717-A as an $N$-acetylmuramoyl-Lalanine amidase was confirmed by LC/MS with the commercially obtained substrate $N$-acetylmuramoyl-Lalanine-D-isoglutamine hydrate (Sigma). A reaction mixture consisting of $100 \mu \mathrm{g}$ rRv3717-A was incubated with $300 \mu \mathrm{g}$ $\mathrm{N}$-acetylmuramoyl-L-alanine-D-isoglutamine hydrate at $37^{\circ} \mathrm{C}$ for $4 \mathrm{~h}$. The reaction was terminated by precipitating the protein with 8.9:1:0.1 acetonitrile:water:formic acid on ice. The samples were centrifuged at $14000 \mathrm{rev} \mathrm{min}^{-1}$ for $10 \mathrm{~min}$ to remove any residual protein and the sample was subjected to analysis on an LC/ESI-MS (Quattro Micro API, Waters) coupled to an UPLC inlet. Briefly, the supernatant was loaded onto an Acquity UPLC BEH shield RP18, $1.7 \mu \mathrm{m}, 2.1 \times$ $100 \mathrm{~mm}$ column comprising a mobile phase of buffer $A$ (acetonitrile, $0.1 \%$ formic acid) and buffer $B$ (water, $0.1 \%$ formic acid) at a flow rate of $0.1 \mathrm{ml} \mathrm{min}{ }^{-1}$. A linear gradient of $5-20 \%$ buffer $B$ was run over $5 \mathrm{~min}$ and the peaks were monitored at $212 \mathrm{~nm}$. MS analysis was carried out with the help of an LCQDeca IonTrap Electrospray Mass Spectrometer (Thermoelectron, Waltham, Massachusetts, USA). The same amount of substrate was incubated in the reaction buffer without protein as a negative control and subjected to LC/MS analysis.

\subsection{Docking of substrate with Rv3717}

Docking of $N$-acetylmuramic acid (NAM) with Rv3717 was performed using the PatchDock server (SchneidmanDuhovny et al., 2005). Coordinates of NAM were obtained from the complex structure of the peptidoglycan-recognition protein in complex with NAM (PDB entry 3olk; Sharma et al., 2012). All parameters used for docking were the defaults of the PatchDock server. The best solution obtained from PatchDock was used for model preparation. All analysis in this study was performed using the same Rv3717-NAM complex structure. A complete peptidoglycan layer (PG) was modelled with Rv3717 using the coordinates of PG from the solution structure of cell-wall peptidoglycan (Meroueh et al., 2006; coordinates received from S. Mobashery, personal communication).

\section{Results}

\subsection{Identification and structure determination of Rv3717}

Domain analysis of all of the proteins in the genome of M. tuberculosis $\mathrm{H} 37 \mathrm{Rv}$ was carried out using the CDD (Marchler-Bauer et al., 2011) and Pfam (Punta et al., 2012) databases. Each of these searches identified two $N$-acetyl- 

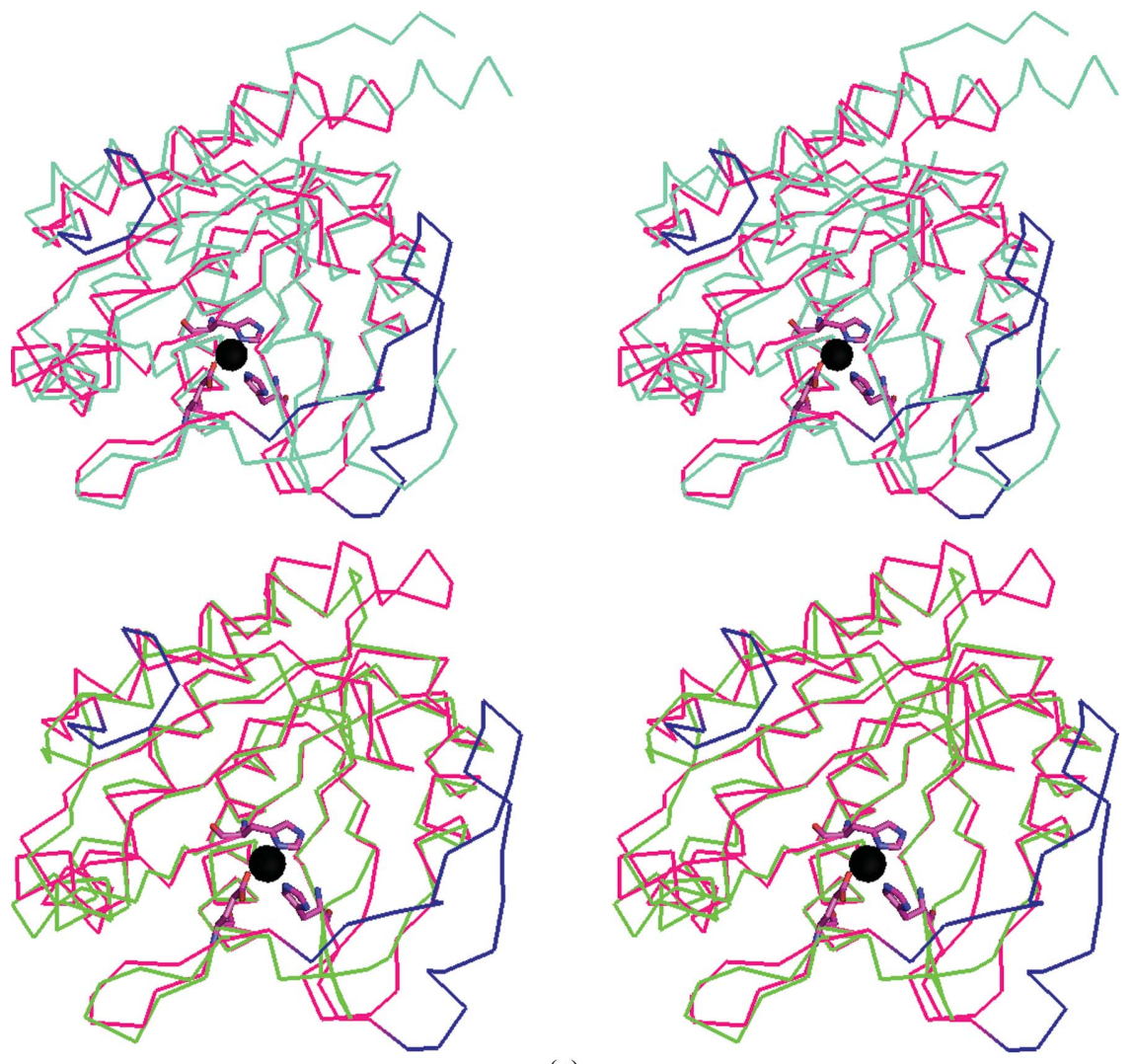

(a)

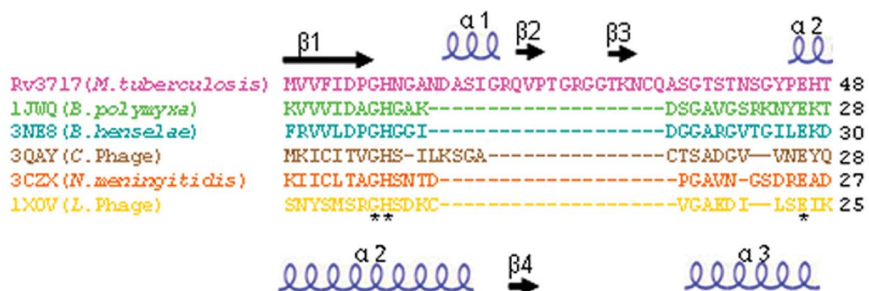

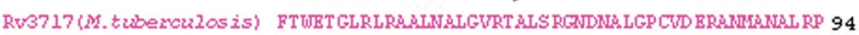
1 JWQ (B. pol Y Y

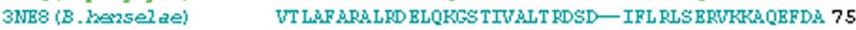
$3 Q j Y$ (C. Phage) 3CZX (N.meningitidis) $1 \times 0 V$ (L. Phage) YNRSLAPVLAD TFRKEGHRUDUII--CPBK-B ERSYKI PRUNSGGY 77 LSQDMRNIVDS ILRNYGLTVKTD GTGK---RMPLRDAVVLIR—GS 70

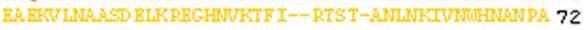

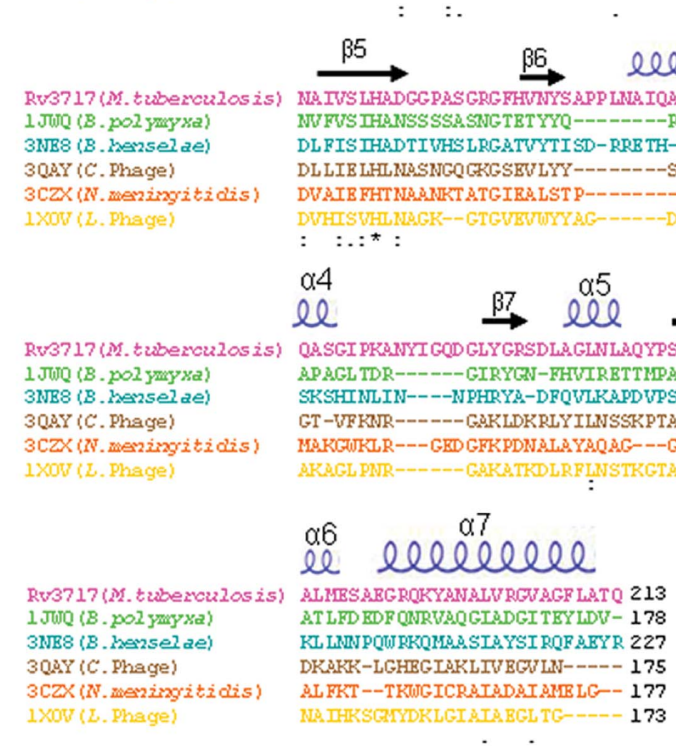

a 4

leblebelel

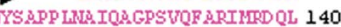

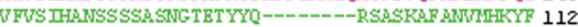
DLFIS IH.DTIVHS LRGATVYTISD-RRB TH- S.FS INR BRNVUSNL 159 DLIB LHLNASNGOGKGSEV LYY--------SNKGELEYATRICDKL 115

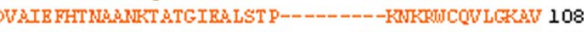
: ** : $\alpha 4$

ll

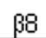
$\alpha 6$ lle DUTRETTMPAVLL BVGILSNA.KBE 152 DFOVLKODDUSVLIBIGYLSNADE 200

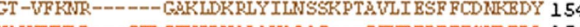

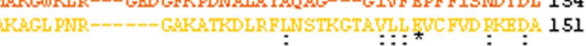

(b)
muramoyl-L-alanine amidases in the M. tuberculosis genome: Rv3915, previously characterized as an $N$-acetylmuramoyl-Lalanine amidase (Deng et al., 2005), and Rv3717, previously annotated as a conserved hypothetical protein. Rv3717 consists of 241 amino acids with a predicted molecular mass of $24.8 \mathrm{kDa}$. The SignalP server (Petersen et al., 2011) predicted the protein to have a signal peptide with a cleavage site between the 24th and 25th residues. Hence, a truncated construct of Rv3717 lacking the predicted signal peptide and starting at the 25th amino-acid position from its $\mathrm{N}$-terminus, comprising 218 residues (including an $\mathrm{N}$-terminal serine as a cloning artefact), was expressed and purified as described in $\$ 2$. The estimated net charge on the protein is $Z=+6$. Purified rRv3717-B was successfully crystallized by the sittingdrop vapour-diffusion method; crystals appeared in 2-3 d. The structure of rRv3717-B was determined to $1.7 \AA$ resolution by the Pt-SAD phasing method using native crystals soaked with a $\mathrm{Pt}$ salt (as described in \$2) and refined to final $R_{\text {work }}$ and $R_{\text {free }}$ values of 0.160 and 0.184 , respectively.

\subsection{Overall structure and role of rRv3717-B as a functional unit}

rRv3717-B represents a unique family of amidases with a single catalytic domain with dimensions of $45 \times 45 \times 38 \AA$. There is one molecule in the asymmetric unit and the protein exists as a monomer. No separate cell-wall/substrate-binding domain (CBD) as present in other amidase crystal structures (Korndörfer et al., 2006) was observed (Fig. 1).

Figure 2

Comparative analysis of Rv3717 with other $\mathrm{N}$ acetylmuramoyl-L-alanine amidases. (a) Stereoview of the superposition of $\mathrm{rRv} 3717-\mathrm{B}$ (magenta) with representative EADs of $N$-acetylmuramoyl-Lalanine amidases from Bartonella henselae (PDB entry 3ne8; cyan) or Bacillus polymxa (PDB entry 1jwq; green) indicates structural similarities (see text for more details). A bound zinc ion (sphere) marks the active site of Rv3717. The ${ }^{16} \beta$-hairpin ${ }^{36}$ at the N-terminus and ${ }^{148}$ loop $^{154}$ of Rv3717 (shown in blue) partially occlude the active site. Active-site residues in $\mathrm{Rv} 3717$ in coordination with $\mathrm{Zn}^{2+}$ are shown in stick representation. (b) Sequence alignment of Rv3717 and its structural homologues as identified from $D A L I$. Sequences are shown in the same colours as their structural counterparts in $(a)$. 
The overall structure of $\mathrm{rRv} 3717-\mathrm{B}$ comprises an $\alpha / \beta$-fold with a central six-stranded twisted $\beta$-sheet surrounded by $\alpha$-helices on both sides (Fig. 1). The final model consists of 213 residues of the molecule. Two residues at the $\mathrm{N}$-terminus (including the additional serine) and three residues at the C-terminus were disordered and could not be included in the final model. One $\mathrm{Zn}^{2+}$ ion was located in the electron-density map in the active site of the metalloenzyme (Fig. 1). A $\beta$-hairpin turn comprising residues 16-36 appears to form a short $\mathrm{N}$-terminal subdomain that limits access to the active site.

\subsection{Structural comparisons}

A search for structural homologues of rRv3717-B was carried out in DALI using the final refined coordinates. The $D A L I$ search resulted in hits for the catalytic or enzymatically active domain (EAD) of autolysins and endolysins. The top hit

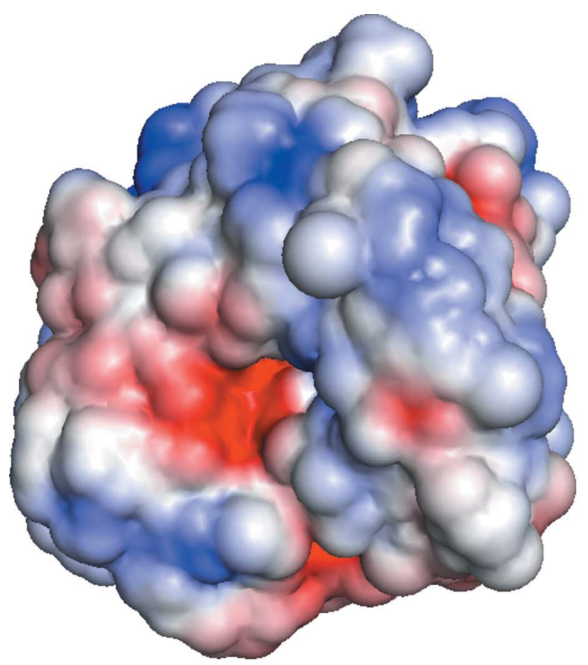

(a)

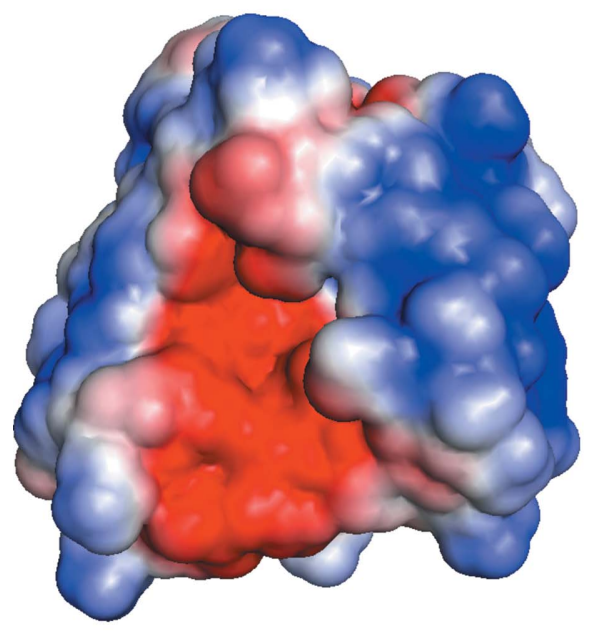

(c)

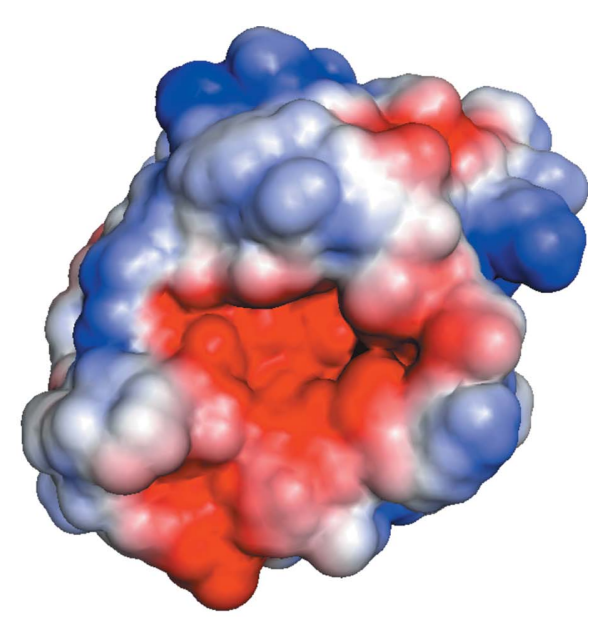

(b)

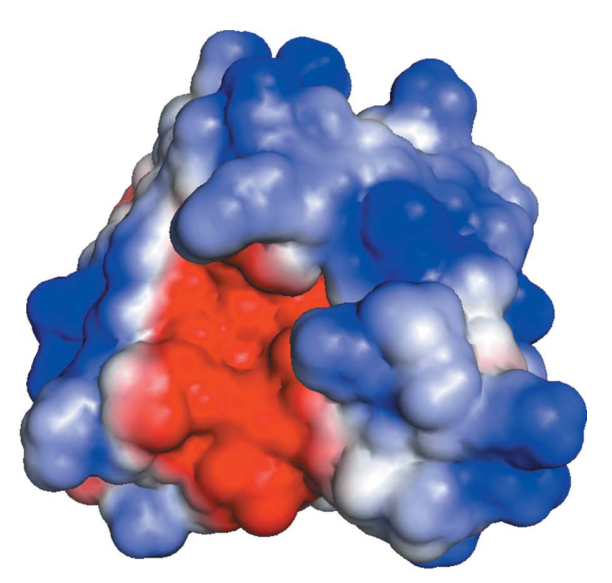

(d)
Figure 3

Electrostatic surface representation of Rv3717 and selected amidases. Molecular-surface representation of $(a)$ rRv3717-B in the same orientation as the EADs of (b) CwlV (PDB entry 1jwq), (c) PlyPSA (PDB entry 1xov) and (d) CD27L (PDB entry 3qay) showing the electrostatic potential displayed on the surface. The electrostatic potential was calculated with $A P B S$ and is coloured with a blue to red gradient from $+2.5 k_{\mathrm{B}} T / \mathrm{e}$ to $-2.5 k_{\mathrm{B}} T / \mathrm{e}$. for rRv3717-B in DALI shows a closest match to PDB entry 1jwq (T. Yamane, Y. Koyama, Y. Nojiri, T. Hikage, M. Akita, A. Suzuki, T. Shirai, F. Ise, T. Shida \& J. Sekiguchi, unpublished work) annotated as an $N$-acetylmuramoyl-L-alanine amidase with a $Z$-score of 25.3 and an r.m.s. deviation of $1.7 \AA$ for $179 \mathrm{C}^{\alpha}$ atoms, suggesting an overall similar structure and a similar amidase role for Rv3717. Other top matches were PDB entries 3ne8 (Yang et al., 2012), 3qay (Mayer et al., 2011), 3czx (Midwest Center for Structural Genomics, unpublished work) and 1 xov (Korndörfer et al., 2006), with Z-scores of 24.3, 21.0, 19.7 and 18.6, respectively. These structures superpose well with rRv3717-B (Figs. $2 a$ and $2 b$ ), with acceptable r.m.s. deviations of $1.6 \AA$ (182 $\mathrm{C}^{\alpha}$ atoms), $2.3 \AA$ (169 $\mathrm{C}^{\alpha}$ atoms), $2.1 \AA$ (166 $\mathrm{C}^{\alpha}$ atoms) and $2.0 \AA$ ( $167 \mathrm{C}^{\alpha}$ atoms), respectively, despite low sequence similarities varying from $13 \%$ for $3 \mathrm{czx}$ to $26 \%$ for 3ne8. Upon closer examination, the overall topology of these structures was found to be identical to Rv3717 except for the insertion of a $\beta$-hairpin comprising residues $16-36$ at the $\mathrm{N}$ terminus (designated ${ }^{16} \beta$-hairpin ${ }^{36}$ ) and an extended loop in the region of 148-154 in rRv3717-B (designated ${ }^{148}$ loop $^{154}$ ) (Fig. 2a). The average $B$ factors of the ${ }^{16} \beta$-hairpin ${ }^{36}\left(22.4 \AA^{2}\right)$ and ${ }^{148} \operatorname{loop}^{154}\left(25.4 \AA^{2}\right)$ are marginally higher than rest of the protein $\left(21.4 \AA^{2}\right)$, suggesting that these regions are more flexible than the rest of the protein.

Electrostatic surface representation of amidases (Mayer et al., 2011) has previously shown that while the active site is negatively charged, the surface leading to the active site is positively charged. The electrostatic surface of rRv3717-B reveals a smaller substratebinding pocket and relatively less negative charge in comparison to other homologues (Fig. 3). The positive charge outside the binding pocket also appears to be distributed all over the surface with no distinct region with a strong positive charge. This unique charge distribution compared with other amidases suggests that the mycobacterial homologue may have evolved a somewhat different mechanism to bind and direct the PG substrate to the active site.

\subsection{Enzymatic activity of Rv3717}

The activity of the single catalytic domain of rRv3717-A was tested by monitoring the decrease in turbidity of a live M. smegmatis suspension as a substrate. Significant lytic activity was observed at 25 and $50 \mu \mathrm{g}$ rRv3717-A 
per millilitre of culture (Fig. $4 a$ ). In comparison, buffer control (no protein) or incubation with BSA resulted in no hydrolysis. The activity of lysozyme towards live M. smegmatis culture was also calculated. The activity of rRv3717-A obtained from this assay was $20.3583 \times 10^{-4} \mathrm{AU} \mathrm{nmol}{ }^{-1} \mathrm{~min}^{-1}$ and was found to be nearly 2.5 -fold higher than that of lysozyme $\left(8.1714 \times 10^{-4} \mathrm{AU} \mathrm{nmol}^{-1} \mathrm{~min}^{-1}\right)$.

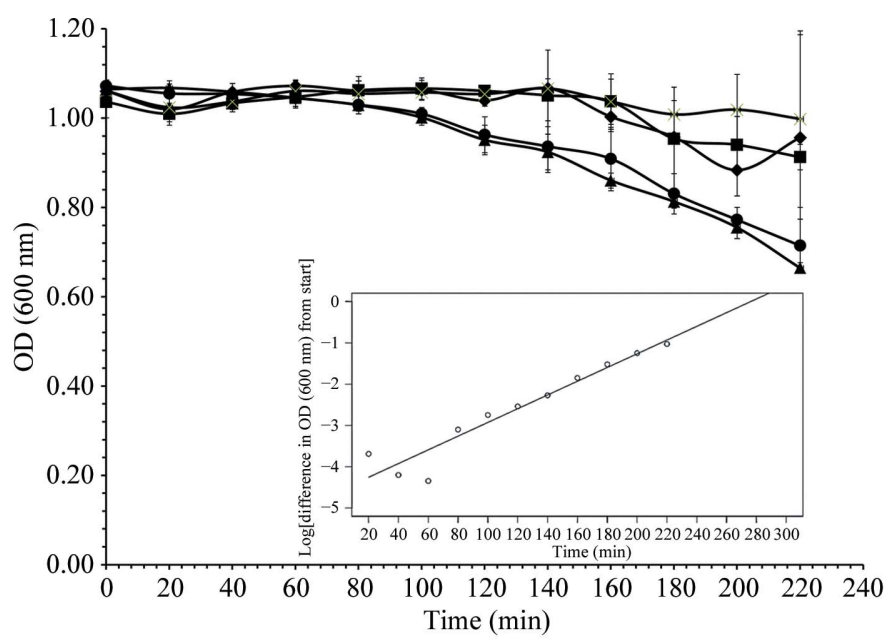

(a)

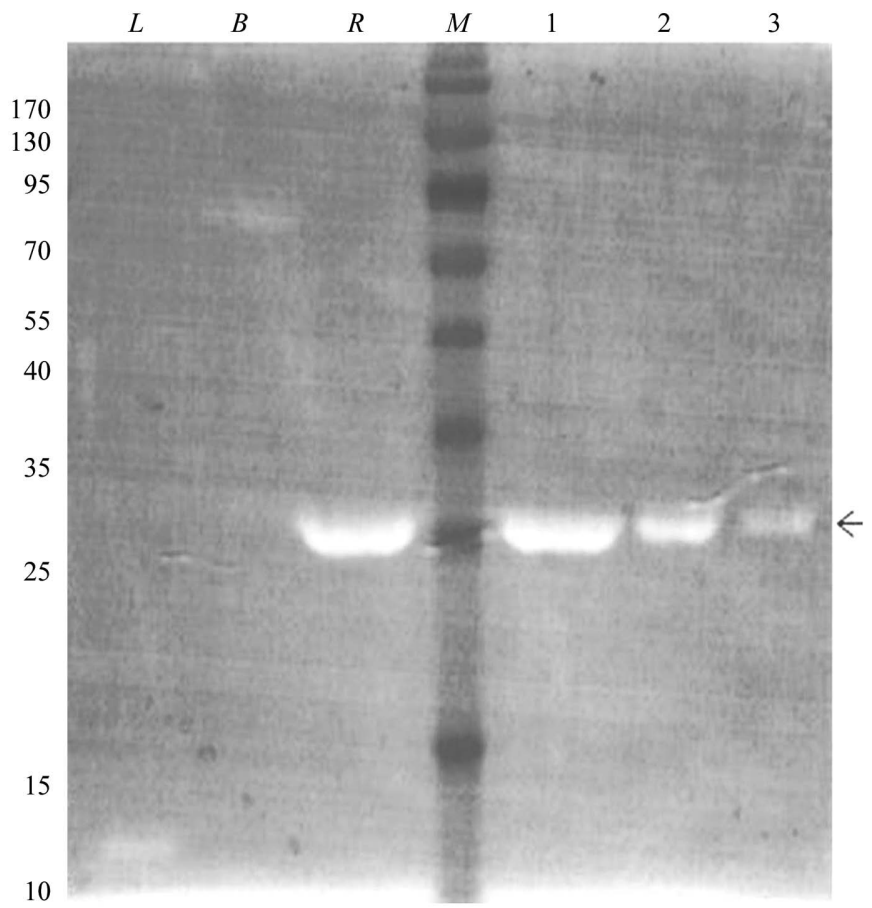

(i)

(b)

The role of rRv3717-A as a general amidase was investigated by zymography using the cell walls of different bacteria (Fig. 4b). rRv3717-A was able to hydrolyse the cell walls of several bacterial species in a dose-dependent manner (i.e. Paenibacillus sp., B. avium, E. coli $\mathrm{DH} 5 \alpha$, E. aerogenes, L. acidophilus, B. thuringiensis, B. pumilus, B. subtilis and E. coli $\mathrm{W} 3110)$, thereby showing that $\mathrm{rRv} 3717-\mathrm{A}$ is a cell-wall hydrolase with broad-spectrum activity.

\subsection{The zinc-binding motif identifies the catalytic site of Rv3717}

$\mathrm{N}$-Acetylmuramoyl-L-alanine amidases are metalloenzymes with a zinc ion being coordinated in the active site of structural homologues (Korndörfer et al., 2006). A metal fluorescence scan using native protein crystals gave an excitation peak at the zinc edge. However, the peak could not unambiguously be assigned to zinc from protein crystals as a peak was also observed in a fluorescence scan with buffer controls. The zinc ion in the active site of rRv3717-B was confirmed by its trigonal bipyramidal coordination geometry in accordance with previously described coordination geometries of $\mathrm{Zn}^{2+}$ (Harding, 2001) as well as by phased anomalous difference maps. The $\mathrm{Zn}$ atom in the crystal structure was found to be coordinated to His11, Glu46 and His101. In the vicinity, three

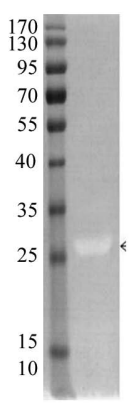

(ii)

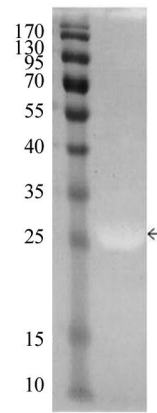

(vi)

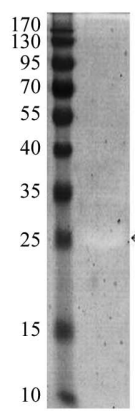

(iii)

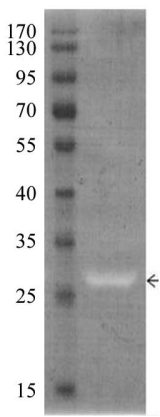

(vii)

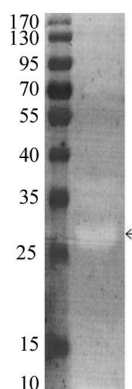

(iv)

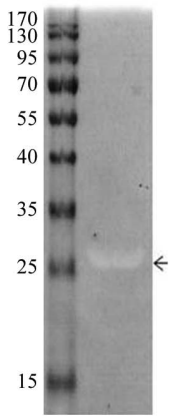

(viii)

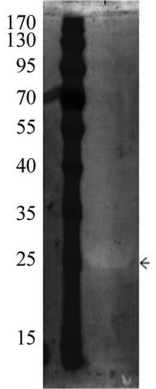

(v)

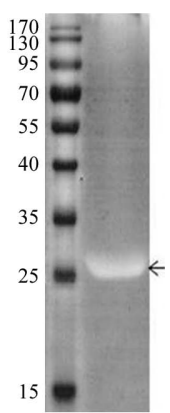

(ix)

\section{Figure 4}

Cell-wall hydrolase activity of Rv3717. (a) The cell-wall hydrolase activity of rRv3717-A was monitored by incubating live M. smegmatis cells with no (filled squares), $1 \mu \mathrm{g} \mathrm{ml}^{-1}$ (crosses), $25 \mu \mathrm{g} \mathrm{ml}^{-1}$ (filled triangles) or $50 \mu \mathrm{g} \mathrm{ml}^{-1}$ (filled circles) rRv3717-A or with BSA (filled diamonds). Error bars represent one standard deviation from the mean value of $\mathrm{OD}_{600}$ in triplicate experiments. The inset shows the mathematical fit for $\mathrm{rRv} 3717-\mathrm{A}$ $\left(50 \mu \mathrm{g} \mathrm{ml}^{-1}\right.$ reaction): $\log \left(\Delta \mathrm{OD}_{600}\right)=0.016 x-4.43$ [general form $\log \left(\Delta \mathrm{OD}_{600}\right)=a x-b$, where the values of $a$ and $b$ are average values for each point]. (b) Zymograph analysis of rRv3717-A with cell suspensions of different heat-killed bacteria as substrates: (i) Paenibacillus sp., (ii) B. avium, (iii) E. coli DH5 $\alpha$, (iv) E. aerogenes, (v) L. acidophilus, (vi) B. thuringiensis, (vii) B. pumilus, (viii) B. subtilis and (ix) E. coli W311. Zymograph analysis was carried out in a dose-dependent manner; in (i) lane 1 contains $4 \mu \mathrm{g}$, lane 2 contains $2 \mu \mathrm{g}$ and lane 3 contains $1 \mu \mathrm{g}$ rRv3717-A. L, Lysozyme (4 $\mu \mathrm{g})$; B, BSA (4 $\mu \mathrm{g}$ ); and $\mathrm{R}, \mathrm{rRv} 3717-\mathrm{A}(4 \mu \mathrm{g})$. Lower doses are not indicated in the other panels for clarity. The zone of clearance indicates cell-wall hydrolysis. A molecularweight marker (lane $M$ ) is included in each panel and is labelled in $\mathrm{kDa}$. 
water molecules are also present to complete its coordination state. This coordination geometry for $\mathrm{Zn}^{2+}$ matches very well with the previously described geometries for $\mathrm{Zn}^{2+}$ in the active site of the structural homologues described above (Korndörfer et al., 2006; Mayer et al., 2011; Yang et al., 2012). In addition, the average $B$ factor for the metal ion refined as $\mathrm{Zn}^{2+}$ $\left(18.5 \AA^{2}\right)$ was found to be close to those of the surrounding residues, confirming it as $\mathrm{Zn}^{2+}$ in the active site of Rv3717. Intriguingly, His11, His101 and Glu46 in the catalytic pocket are among the only conserved residues in the sequence alignment of Rv3717 with its structural homologues (Fig. 2b), emphasizing the role of these conserved residues in zinc binding and catalysis despite significant sequence divergence. Gly10, which has a structural role, and Glu176, which indirectly interacts with $\mathrm{Zn}^{2+}$ through a water-mediated interaction, are the only other conserved residues in the sequence alignment.

The zinc ion seems to bind preferentially to the protein, as no extraneous $\mathrm{Zn}^{2+}$ was added either in protein preparation or during crystallization. The intrinsically bound $\mathrm{Zn}^{2+}$ ion also enabled hydrolytic activity towards heat-killed M. smegmatis cells even when no additional $\mathrm{Zn}^{2+}$ was added to the reaction mixture (Fig. 5). The lytic activity increased marginally with increasing amounts of $\mathrm{Zn}^{2+}$. Despite the apparent preference for $\mathrm{Zn}^{2+}$ binding, several other divalent ions that were tested $\left(\mathrm{Cu}^{2+}, \mathrm{Co}^{2+}\right.$ and $\left.\mathrm{Mg}^{2+}\right)$ appeared to substitute for $\mathrm{Zn}^{2+}$ and achieved similar levels of lytic activity with increasing concentrations of the respective metal ion as observed for $\mathrm{Zn}^{2+}$ (Supplementary Fig. S1 ${ }^{\mathbf{1}}$ ).

\subsection{Analysis of enzymatic products of Rv3717 activity by LC/MS}

The enzymatic products obtained from the activity of rRv3717-A were analyzed to confirm the role of the enzyme as an amidase using $N$-acetylmuramoyl-L-alanyl-D-isoglutamate as a substrate (Fig. 6a). Upon digestion by rRv3717-A, two peaks corresponding to the digested products L-alanyl-Disoglutamine (2.25 $\mathrm{min})$ and $\mathrm{N}$-acetylmuramic acid (2.72 $\mathrm{min})$ were obtained from HPLC separation (Fig. 6b). Massspectrometric analysis confirmed the $2.25 \mathrm{~min}$ peak to be L-alanyl-D-isoglutamine $(\mathrm{m} / \mathrm{z}=218.26)$ and the $2.72 \mathrm{~min}$ peak to be $N$-acetylmuramic acid $(\mathrm{m} / \mathrm{z}=294.27)$ (Fig. $6 \mathrm{c})$. None of the other potential cleavage products $\mathrm{N}$-acetylmuramoylL-alanine, L-alanine or D-isoglutamate were observed, suggesting the highly specific nature of catalysis of rRv3717-A. Furthermore, MS/MS analysis of the substrate and products was performed to confirm the identity of the masses obtained in the spectrum (Supplementary Fig. S2). In addition, a control reaction with no protein but only $N$-acetylmuramoyl-L-alanylD-isoglutamate in the reaction mixture yielded only the original substrate with an $\mathrm{m} / \mathrm{z}$ of 493.06. No detectable masses for D-isoglutamine, L-alanine or $\mathrm{N}$-acetylmuramoyl-L-alanine were identified, confirming that the products result from

\footnotetext{
${ }^{1}$ Supplementary material has been deposited in the IUCr electronic archive (Reference: LV5048).
}

enzymatic activity of rRv3717-A and not from spontaneous or random hydrolysis (Supplementary Fig. S3).

\subsection{Modelling of Rv3717 with cell-wall peptidoglycan}

In order to gain insights into the mode of PG binding to rRv3717-B, a blind docking experiment using the PatchDock server (Schneidman-Duhovny et al., 2005) was first carried out using $N$-acetylmuramic acid (NAM) as a potential ligand. The top results from the server revealed that NAM is bound in the active site of rRv3717-B close to the zinc metal ion on one side and a hydrophobic region on the other, thereby confirming the zinc-binding pocket as the substrate-binding site (Fig. 7a). Zinc is present on one of the walls of the active site, allowing substrate to bind in this pocket. This docked position of NAM obtained from the PatchDock server was used to model the interactions of Rv3717 with the peptidoglycan layer (Fig. 7b). The electrostatic surface of the protein showed that the catalytic centre where NAM is present is rich in negative charge (Fig. 3) and is surrounded by a hydrophobic surface, suggesting that the PG substrate binds in the pocket near to the zinc motif through hydrophobic interactions. The labile amide bond on the PG layer is pushed into the electronegative active site where catalysis takes place through a proton-relay system.

The active site of Rv3717 is partially occluded by a $\beta$-hairpin insertion in the $\mathrm{N}$-terminus of $\mathrm{rRv} 3717-\mathrm{B}$ and may restrict the access of substrate to the catalytic centre from one side. We looked at other potential interactions when the amidase comes into contact with the substrate PG layer and found that the nature of several conserved residues of other amidases is modified in Rv3717. For instance, Leu98 of CD27L (Mayer et al., 2011) is a conserved hydrophobic residue: it is Trp in PlyPSA (Korndörfer et al., 2006) and Tyr in AmiB (Yang et al., 2012) and CwlV (Shida et al., 2001). However, this

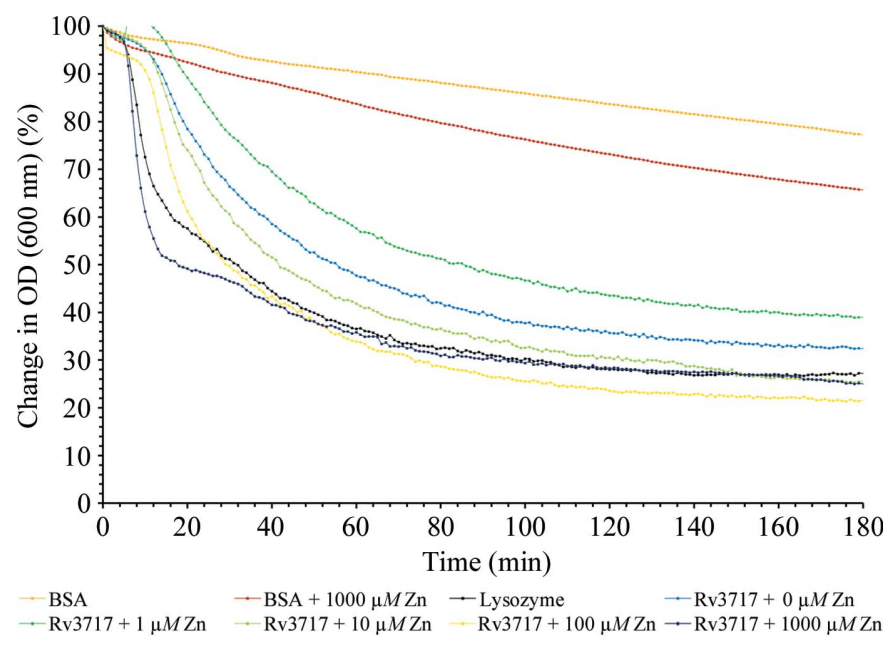

\section{Figure 5}

Effect of metal ions on the activity of rRv3717-A. Activity of rRv3717-A towards heat-killed $M$. smegmatis estimated with no (blue), $1 \mu \mathrm{m}$ (sea green), $10 \mu \mathrm{m}$ (light green), $100 \mu \mathrm{m}$ (yellow) or $1000 \mu \mathrm{m}$ (brown) $\mathrm{Zn}^{2+}$ at $37^{\circ} \mathrm{C}$. BSA (orange) or BSA $+1000 \mu \mathrm{m} \mathrm{Zn}^{2+}$ (red) were used as negative controls, while lysozyme (black) served as a positive control for the reaction. 
position is occupied by Asn114 in Rv3717, suggesting that conservation of a hydrophobic residue at this position is not necessary for the activity of Rv3717. Additional conserved residues suggested to be involved in substrate binding, namely Leu130, Tyr131 and Ile132 of the Clostridium difficile amidase CD27L (Mayer et al., 2011), are Leu127, Arg128 and Phe129 in PlyPSA (Korndörfer et al., 2006), Phe128, His129 and Val130 in CwlV (PDB entry 1jwq) and Phe351, Gln352 and Val353 in AmiB (PDB entry 3ne8). These residues are Leu162, Ala163 and Gly164 in Rv3717 and are aligned for potential interactions with PG in the model (Fig. $7 b$ ). The less bulky side chains in Rv3717 in this region appear to enlarge the substrate-binding cavity and suggest that Rv3717 may have evolved independent and altered modes of substrate recognition and binding.

\section{Discussion}

Rv3717 is a unique autolysin as it lacks the cell-wall-binding domain (CBD) that has the propensity to bind to fibronectin and laminin (Kumar et al., 2013). The cell-wall hydrolases of various phages are known to exhibit high specificity towards the cell walls of host bacteria. This specificity is rendered to the hydrolase by unique structural elements in the CBD

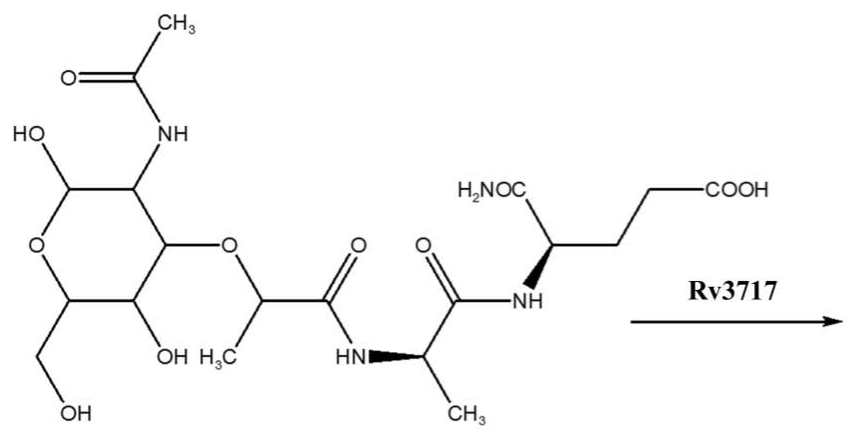

$\mathrm{N}$-Acetyl-L-alanyl-D-isoglutamine (M)

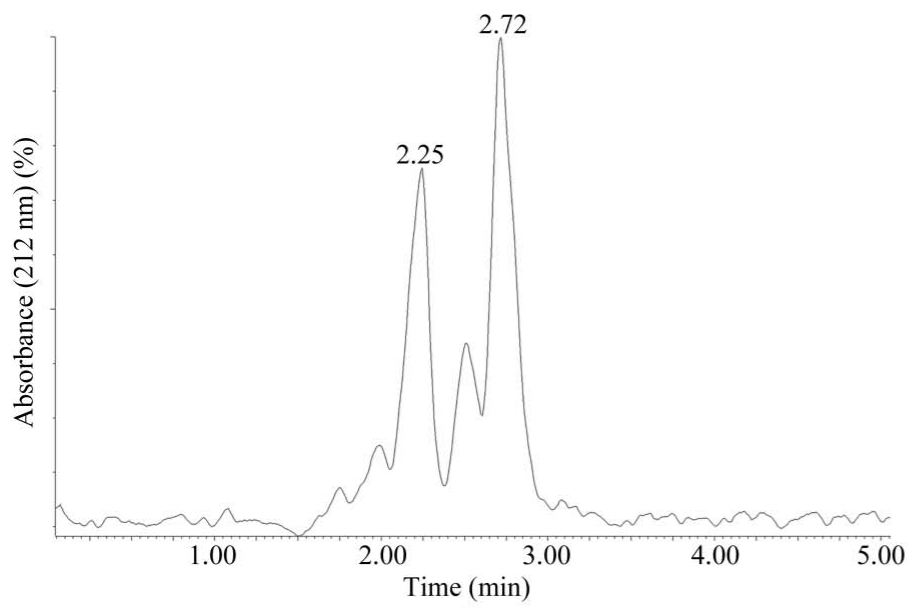

(b)
Figure 6

LC/MS analysis. (a) Schematic representation of the enzymatic reaction of Rv3717 towards $N$-acetylmuramoyl-L-alanyl-D-isoglutamine (M) resulting in products $\mathrm{M}_{1}$ (muramic acid; molecular mass 293.27 Da) and $\mathrm{M}_{2}$ (L-alanyl-D-isoglutamine (molecular mass $217.26 \mathrm{Da}$ ), which were analyzed by LC/MS. (b) The reaction mixture was purified by ultrahighpressure liquid chromatography (UPLC) into separate peaks of L-alanylD-isoglutamine $(2.25 \mathrm{~min})$ and muramic acid $(2.72 \mathrm{~min})$. (c) MS analysis of the UPLC-separated peaks corresponding to L-alanyl-D-isoglutamine ( $2.25 \mathrm{~min}$; top panel) and muramic acid (2.72 $\mathrm{min}$; bottom panel).

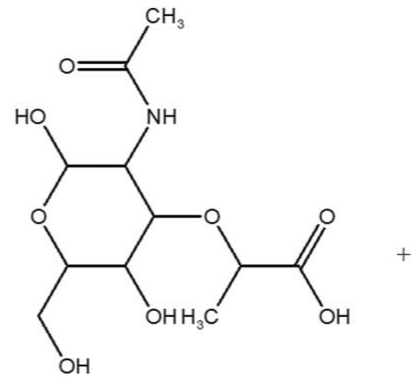

$N$-Acetylmuramic acid $\left(\mathrm{M}_{1}\right)$

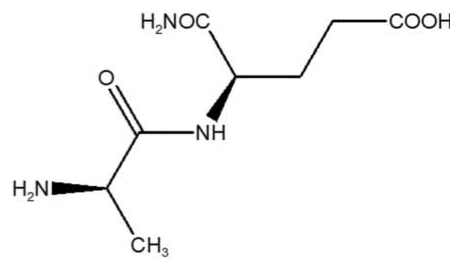

L-Alanyl-D-isoglutamine $\left(\mathrm{M}_{2}\right)$ (a)
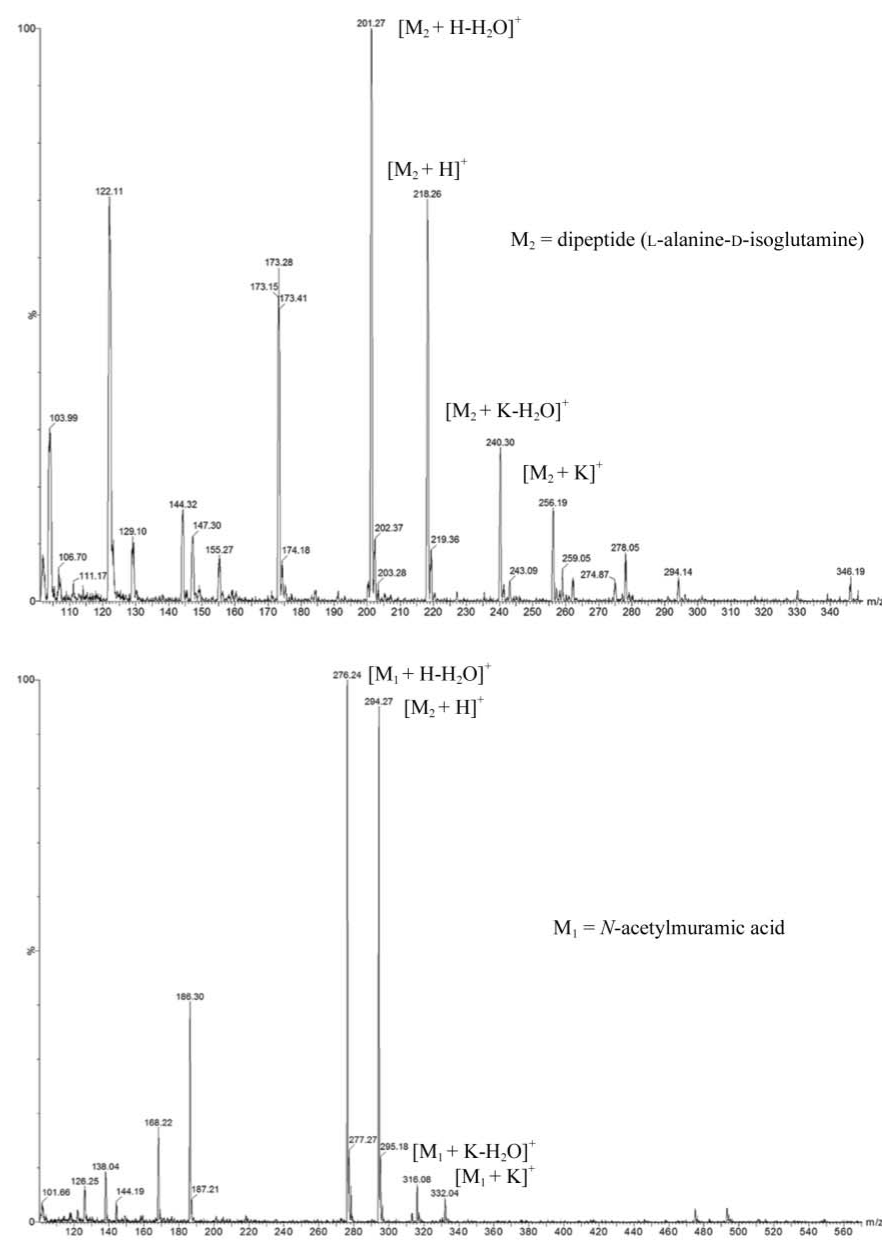

(c) 
independently of the catalytic activity. In the absence of a $\mathrm{CBD}$, it remains intriguing how $\mathrm{Rv} 3717$ binds to a wide spectrum of substrates to perform its role in cell-wall hydrolysis. The cell walls of bacteria carry a weak negative charge and the binding of the substrate cell wall is thus affected by the net charge on the CBD (Low et al., 2011). In the absence of the $\mathrm{CBD}$, the catalytic domain of $B$. anthracis amidase, PlyL, shows high lytic activity towards a broader host range than the full-length protein (Low et al., 2005). This ability of the EAD of PlyL to be catalytically active has been postulated to be a consequence of the net positive charge of $Z=+6$ on the protein. Engineering of net charge on the catalytic domain of XlyA, a $B$. subtilis amidase, from $Z=-3$ to $Z=+3$ converts the EAD from an inactive to an active state (Low et al., 2011), confirming that EADs with low net positive charge are catalytically active on their own.

Rv3717 thus appears to belong to a new subclass of autolysins that have evolved without a CBD and perform their function independently in a nonspecific manner. The single EAD domain of Rv3717 overcomes the absence of a CBD through a net positive charge of $Z=+6$ on the protein. The absence of a CBD, however, may lead to low processivity of the enzyme upon cleavage of the dipeptide and release of the substrate. Rv3717 appears to counter this by having a relatively more hydrophobic surface compared with other

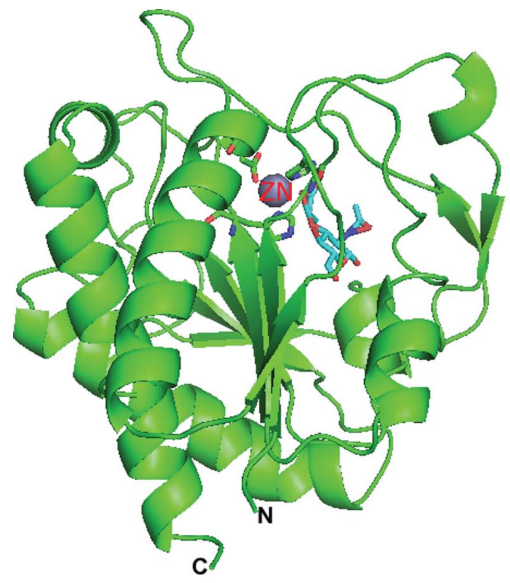

(a)
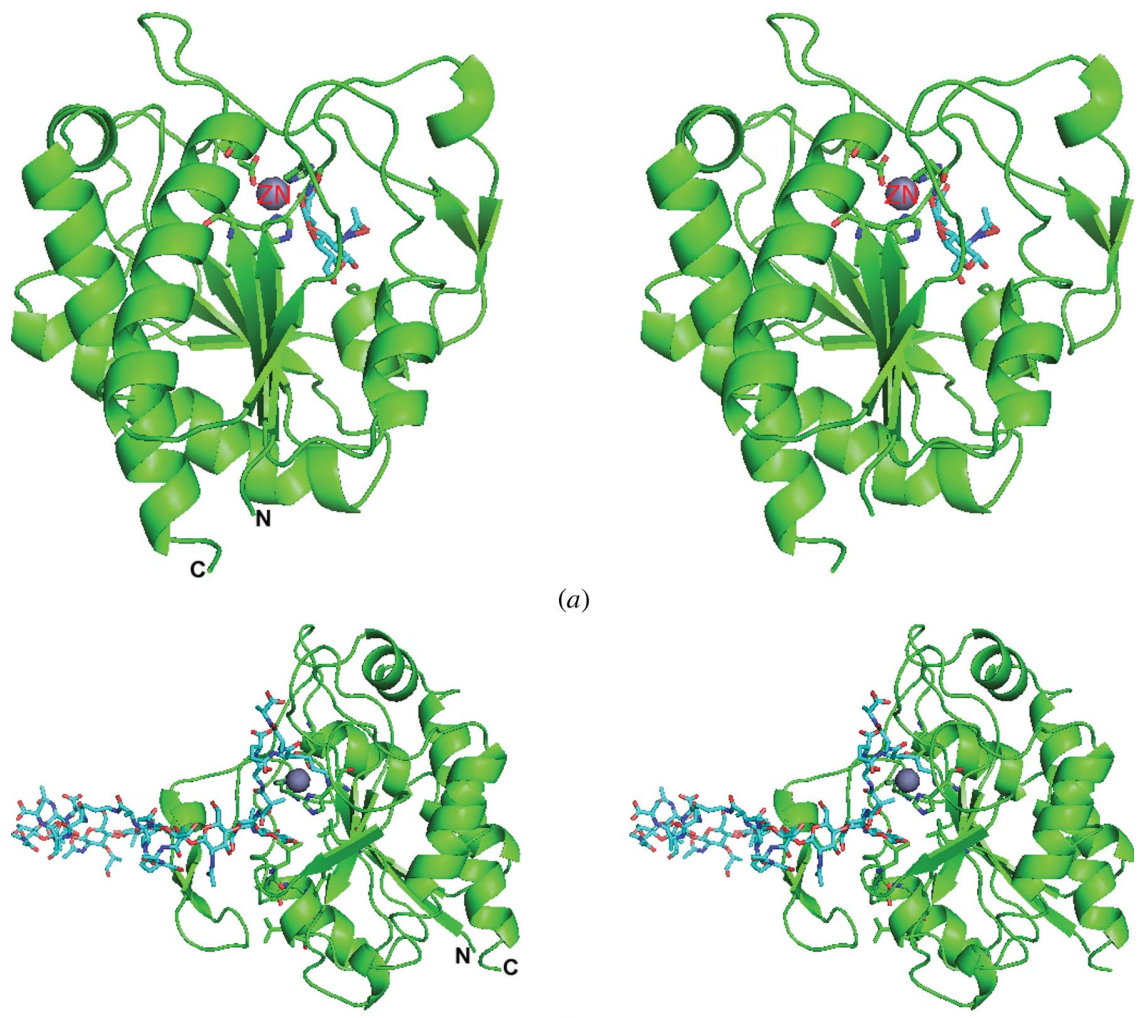

(b)

Figure 7

Proposed model of Rv3717 with cell-wall peptidoglycan. (a) Stereoview of Rv3717 in complex with $\mathrm{N}$-acetylmuramic acid as obtained from the PatchDock server. (b) Stereoview of a proposed model of Rv3717 with cell-wall peptidoglycan. The hydrophobic residues that may be involved in PG binding as well as the catalytic residues of Rv3717 are shown in stick representation. hydrolases (Fig. 3). The distributed positive charge on the surface of Rv3717 enables interactions with multiple regions of the substrate cell-wall peptidoglycan. These adaptations appear to help the enzyme perform its function in an effective manner, as shown by the nearly 2.5 -fold higher specific activity of Rv3717 compared with lysozyme. In addition, the model of Rv3717 with peptidoglycan helps to identify several residues involved in hydrophobic interactions with the substrate to carry out its function. Conformational changes in Rv3717 upon substrate binding may lead to changes in the surface to enable more residues to interact with the substrate cell wall during the catalytic cycle.

Apart from the utilization of a net positive charge in substrate binding, Rv3717 appears to have evolved a mechanism of autoregulation through a unique short hairpin loop that is absent in other homologues. An autoregulatory mechanism has been observed in a few other PG hydrolases [e.g. M. tuberculosis RipA (Ruggiero et al., 2010; Böth et al., 2011), Staphylococcus aureus LytM (Odintsov et al., 2004) and Streptococcus pneumoniae LytC (Pérez-Dorado et al., 2010)]. An autoregulatory mechanism through conformational changes has also been observed in $\mathrm{N}$-acetylmuramoyl-Lalanine amidases of both the Amidase_2 (comprising AmiD and AmpD; Carrasco-Lopez et al., 2011) and the Amidase_3 (comprising AmiA, AmiB and AmiC; van Heijenoort, 2011) families. However, both these families differ from each other in sequence and structure (Vollmer et al., 2008).

The autoregulatory amphipathic helix of AmiB is present in an analogous position to the autoregulatory $\beta$-hairpin of $\operatorname{Rv} 3717$. Asp259 of the autoregulatory helix makes direct interactions with the zinc ion in $\mathrm{AmiB}$, while another residue, Asp290, extends into the active site, thereby occluding the active site. Unlike the $\alpha$-helix of AmiB, however, the $\beta$-hairpin of $\operatorname{Rv} 3717$ appears to be in an open conformation as it does not make any interactions with $\mathrm{Zn}^{2+}$ or the active-site residues. Moreover, NAM in our proteinligand model (Fig. 7a) binds in the position occupied by the helix of AmiB. Rv3717 contains another short loop, comprising residues Ala148, Asn149, Tyr150, Ile151, Gly152, Gln153 and Asp154, that was not observed in its structural homologues. This loop is present in an equivalent position to that occupied by Asn343 and Asn344 of AmiB. In the AmiB structure Asn344 is positioned to make potential interactions with the occluding helix through watermediated interactions. It is provocative to suggest a similar critical role in autoregulation for the ${ }^{148}$ loop $^{154}$ in Rv3717. Although no clear interactions are observed for either the $\beta$-hairpin or the ${ }^{148}$ loop $^{154}$ in the present 'open' conformation of Rv3717, they may be 
possible in its 'closed' state to regulate activity during different growth stages of the mycobacterium.

\section{Conclusion}

In conclusion, this is the first report of the structure of an $\mathrm{N}$-acetylmuramoyl-L-alanine amidase from M. tuberculosis. Rv3717 represents a new family of lytic amidases that do not have a separate $\mathrm{CBD}$ and are regulated conformationally, analogous to the cell-division amidase AmiB (Yang et al., 2012). The enzymatic products of Rv3717 were analyzed by LC/MS to confirm its activity. A net positive charge on the protein, combined with the absence of a CBD, enables broad substrate specificity for Rv3717, as shown by zymography using the cell walls of several heat-killed bacteria. The surface representations suggest that the rim of the binding pocket of the enzyme is hydrophobic, in contrast to the available structures from other eubacteria. Whether this difference in surface charge is an artefact of local conformations of the apo enzyme in the 'open' conformation or whether the mycobacterial enzyme has evolved for the recognition of specific cell membranes of a few bacteria needs to be investigated further.

We thank Dr Rakesh Sharma and Dr Shantanu Chowdhury for evaluation of the manuscript and Dr Lipi Thukral for discussions. We thank Dr Rajesh Gokhale for his support for the project, Professor Stewart Shuman and Dr Krishna Murari Sinha for the pET-28-His10-Smt3 plasmid and Shahriar Mobashery for sharing the coordinates of peptidoglycan. The help and suggestions of Seema Joshi and Dr Santosh Pasha in the LC/MS experiments are gratefully acknowledged. We acknowledge the help rendered by Dr Hassan Belrhali and Dr Babu Manjashetty during data collection on the BM-14 beamline of ESRF and by Maurizio Maurizio Polentarutti and Nicola Demitri during metal-ion scans at Sincrotrone Trieste. This work was supported by the Council of Scientific and Industrial Research Project Grant FAC-02 (BT) and BSC0121 (SR). SK, DK and AM acknowledge CSIR, while AK and PS acknowledge ICMR for Senior Research Fellowships. Funding for data collection on the BM14 beamline of ESRF by the Department of Biotechnology (India) and at Sincrotrone Trieste by the Department of Science and Technology (India) is acknowledged.

\section{References}

Allignet, J., Aubert, S., Dyke, K. G. \& El Solh, N. (2001). Infect. Immun. 69, 712-718.

Baker, N. A., Sept, D., Joseph, S., Holst, M. J. \& McCammon, J. A. (2001). Proc. Natl Acad. Sci. USA, 98, 10037-10041.

Berry, A. M., Lock, R. A., Hansman, D. \& Paton, J. C. (1989). Infect. Immun. 57, 2324-2330.

Bhardwaj, A. et al. (2011). Tuberculosis, 91, 479-486.

Böth, D., Schneider, G. \& Schnell, R. (2011). J. Mol. Biol. 413, 247-260.

Brennan, P. J. \& Nikaido, H. (1995). Annu. Rev. Biochem. 64, 29-63. Bricogne, G., Blanc, E., Brandl, M., Flensburg, C., Keller, P., Paciorek, W., Roversi, P., Sharff, A., Smart, O. S., Vonrhein, C. \& Womack, T. O. (2011). BUSTER v.2.10.0. Cambridge: Global Phasing Ltd.
Buist, G., Kok, J., Leenhouts, K. J., Dabrowska, M., Venema, G. \& Haandrikman, A. J. (1995). J. Bacteriol. 177, 1554-1563.

Carrasco-López, C., Rojas-Altuve, A., Zhang, W., Hesek, D., Lee, M., Barbe, S., André, I., Ferrer, P., Silva-Martin, N., Castro, G. R., Martínez-Ripoll, M., Mobashery, S. \& Hermoso, J. A. (2011). J. Biol. Chem. 286, 31714-31722.

Chen, V. B., Arendall, W. B., Headd, J. J., Keedy, D. A., Immormino, R. M., Kapral, G. J., Murray, L. W., Richardson, J. S. \& Richardson, D. C. (2010). Acta Cryst. D66, 12-21.

Deng, L. L., Humphries, D. E., Arbeit, R. D., Carlton, L. E., Smole, S. C. \& Carroll, J. D. (2005). Biochim. Biophys. Acta, 1747, 57-66.

Emsley, P., Lohkamp, B., Scott, W. G. \& Cowtan, K. (2010). Acta Cryst. D66, 486-501.

Engh, R. A. \& Huber, R. (1991). Acta Cryst. A47, 392-400.

Evans, G. \& Pettifer, R. (2001). J. Appl. Cryst. 34, 82-86.

Harding, M. M. (2001). Acta Cryst. D57, 401-411.

Heijenoort, J. van (2011). Microbiol. Mol. Biol. Rev. 75, 636-663.

Hett, E. C. \& Rubin, E. J. (2008). Microbiol. Mol. Biol. Rev. 72, $126-156$.

Hoffmann, C., Leis, A., Niederweis, M., Plitzko, J. M. \& Engelhardt, H. (2008). Proc. Natl Acad. Sci. USA, 105, 3963-3967.

Jayaswal, R. K., Lee, Y.-I. \& Wilkinson, B. J. (1990). J. Bacteriol. 172, 5783-5788.

Kaur, D., Berg, S., Dinadayala, P., Gicquel, B., Chatterjee, D., McNeil, M. R., Vissa, V. D., Crick, D. C., Jackson, M. \& Brennan, P. J. (2006). Proc. Natl Acad. Sci. USA, 103, 13664-13669.

Korndörfer, I. P., Danzer, J., Schmelcher, M., Zimmer, M., Skerra, A. \& Loessner, M. J. (2006). J. Mol. Biol. 364, 678-689.

Kumar, A., Saigal, K., Malhotra, K., Sinha, K. M. \& Taneja, B. (2011). J. Biol. Chem. 286, 19652-19661.

Kumar, S., Puniya, B., Parween, S., Nahar, P. \& Ramachandran, S. (2013). PLoS One, 8, e69790.

Leslie, A. G. W. \& Powell, H. R. (2007). Evolving Methods for Macromolecular Crystallography, edited by R. J. Read \& J. L. Sussman, pp. 41-51. Dordrecht: Springer.

Li, Z. S., Beveridge, T. J., Betts, J. \& Clarke, A. J. (1999). Microbiology, 145, 169-176.

Loessner, M. J., Kramer, K., Ebel, F. \& Scherer, S. (2002). Mol. Microbiol. 44, 335-349.

Lopez-Marin, L. M. (2012). Clin. Dev. Immunol. 2012, 917860.

Low, L. Y., Yang, C., Perego, M., Osterman, A. \& Liddington, R. C. (2005). J. Biol. Chem. 280, 35433-35439.

Low, L. Y., Yang, C., Perego, M., Osterman, A. \& Liddington, R. (2011). J. Biol. Chem. 286, 34391-34403.

Marchler-Bauer, A. et al. (2011). Nucleic Acids Res. 39, D225-D229.

Mayer, M. J., Garefalaki, V., Spoerl, R., Narbad, A. \& Meijers, R. (2011). J. Bacteriol. 193, 5477-5486.

McNeil, M. R. \& Brennan, P. J. (1991). Res. Microbiol. 142, 451-463.

Meroueh, S. O., Bencze, K. Z., Hesek, D., Lee, M., Fisher, J. F., Stemmler, T. L. \& Mobashery, S. (2006). Proc. Natl Acad. Sci. USA, 103, 4404-4409.

Milohanic, E., Jonquières, R., Cossart, P., Berche, P. \& Gaillard, J.-L. (2001). Mol. Microbiol. 39, 1212-1224.

Murshudov, G. N., Skubák, P., Lebedev, A. A., Pannu, N. S., Steiner, R. A., Nicholls, R. A., Winn, M. D., Long, F. \& Vagin, A. A. (2011). Acta Cryst. D67, 355-367.

Nguyen, L. \& Thompson, C. J. (2006). Trends Microbiol. 14, 304-312.

Odintsov, S. G., Sabala, I., Marcyjaniak, M. \& Bochtler, M. (2004). J. Mol. Biol. 335, 775-785.

Painter, J. \& Merritt, E. A. (2006). Acta Cryst. D62, 439-450.

Panjikar, S., Parthasarathy, V., Lamzin, V. S., Weiss, M. S. \& Tucker, P. A. (2005). Acta Cryst. D61, 449-457.

Panjikar, S., Parthasarathy, V., Lamzin, V. S., Weiss, M. S. \& Tucker, P. A. (2009). Acta Cryst. D65, 1089-1097.

Pérez-Dorado, I., González, A., Morales, M., Sanles, R., Striker, W., Vollmer, W., Mobashery, S., García, J. L., Martínez-Ripoll, M., García, P. \& Hermoso, J. A. (2010). Nature Struct. Mol. Biol. 17, 576-581. 
Petersen, T. N., Brunak, S., von Heijne, G. \& Nielsen, H. (2011). Nature Methods, 8, 785-786.

Potterton, E., Briggs, P., Turkenburg, M. \& Dodson, E. (2003). Acta Cryst. D59, 1131-1137.

Punta, M. et al. (2012). Nucleic Acids Res. 40, D290-D301.

Raviglione, M., Marais, B., Floyd, K., Lönnroth, K., Getahun, H., Migliori, G. B., Harries, A. D., Nunn, P., Lienhardt, C., Graham, S., Chakaya, J., Weyer, K., Cole, S., Kaufmann, S. H. \& Zumla, A. (2012). Lancet, 379, 1902-1913.

Ruggiero, A., Marasco, D., Squeglia, F., Soldini, S., Pedone, E., Pedone, C. \& Berisio, R. (2010). Structure, 18, 1184-1190.

Rupp, M. E., Fey, P. D., Heilmann, C. \& Götz, F. (2001). J. Infect. Dis. 183, 1038-1042.

Schneidman-Duhovny, D., Inbar, Y., Nussinov, R. \& Wolfson, H. J. (2005). Nucleic Acids Res. 33, W363-W367.

Sharma, P., Yamini, S., Dube, D., Singh, A., Sinha, M., Dey, S., Mitra,
D. K., Kaur, P., Sharma, S. \& Singh, T. P. (2012). J. Biol. Chem. 287, $22153-22164$.

Shida, T., Hattori, H., Ise, F. \& Sekiguchi, J. (2001). J. Biol. Chem. 276, 28140-28146.

Smith, T. J., Blackman, S. A. \& Foster, S. J. (2000). Microbiology, 146, 249-262.

Vollmer, W., Blanot, D. \& de Pedro, M. A. (2008). FEMS Microbiol. Rev. 32, 149-167.

World Health Organization (2011). Global Tuberculosis Control. Geneva: World Health Organization. http://whqlibdoc.who.int/ publications/2011/9789241564380_eng.pdf.

Winn, M. D. et al. (2011). Acta Cryst. D67, 235-242.

Yang, D. C., Tan, K., Joachimiak, A. \& Bernhardt, T. G. (2012). Mol. Microbiol. 85, 768-781.

Zuber, B., Chami, M., Houssin, C., Dubochet, J., Griffiths, G. \& Daffé, M. (2008). J. Bacteriol. 190, 5672-5680. 